\title{
ON THE TRACE OF AN IDEMPOTENT IN A GROUP RING
}

\author{
GERALD H. CLIFF AND SUDARSHAN K. SEHGAL
}

\begin{abstract}
Let $K G$ be the group ring of a polycyclic by finite group $G$ over a field $K$ of characteristic zero. It is proved that if $e=\sum e(g) g$ is a nontrivial idempotent in $K G$ then its trace $e(1)$ is a rational number $r / s,(r, s)=1$, with the property that for every prime divisor $p$ of $s, G$ has an element of order $p$. This result is used to prove that if $R$ is a commutative ring of characteristic zero, without nontrivial idempotents and $G$ is a polycyclic by finite group such that no group order $\neq 1$ is invertible in $R$, then $R G$ has no nontrivial idempotents.
\end{abstract}

1. Let $K G$ be the group ring of a group $G$ over a field $K$. By the trace of an element $\alpha=\Sigma_{g} \alpha(g) g$ of $K G$ is understood $\alpha(1)$, the coefficient of the identity in $\alpha$. The following two statements regarding the trace of an idempotent in $K G$ are well known.

TheOREM (ZALESSKII [7]). The trace of an idempotent in $K G$ lies in the prime subfield of $K$.

THEOREM (KAPLANSKY, SEE [4]). If $K$ is a field of characteristic zero, the trace of a nontrivial idempotent in $K G$ lies strictly between 0 and 1 .

We expect that in the characteristic zero case one should be able to say more, namely the denominator of the trace of a nontrivial idempotent is a $|G|$ number, in the sense that for every prime $p$ dividing this denominator, $G$ has an element of order $p$. This statement is proved in Theorem 1 for polycyclic by finite groups. We apply this to prove that if $R$ is a unital commutative ring of characteristic zero without nontrivial idempotents, with the property that no group element $\neq 1$ has order invertible in $R$ and $G$ is polycyclic by finite, then $R G$ has no nontrivial idempotents. This is proved for supersolvable groups in [3] and [5].

\section{Results.}

THEOREM 1. Let $K G$ be the group ring of a polycyclic by finite group $G$ over a field $K$ of characteristic zero. Let $e=\Sigma_{g} e(g) g$ be a nontrivial idempotent. Write

Received by the editors March 20, 1976.

AMS (MOS) subject classifications (1970). Primary 16A26; Secondary 20 C05.

Key words and phrases. Group rings, idempotent, trace. 
$e(1)=r / s$ with $(r, s) \doteq 1$. If a prime $p$ divides $s$, then there exists $1 \neq g \in G$ of p-power order with $e(g) \neq 0$.

THEOREM 2. Let $R G$ be the group ring of a polycyclic by finite group $G$ over a commutative unital ring $R$ of characteristic zero. Suppose that $R$ has no nontrivial idempotents and that no group element $\neq 1$ has order invertible in $R$. Then $R G$ has no nontrivial idempotents.

3. Proof of Theorem 1. Denoting conjugate elements $g$ and $h$ of $G$ by $g \sim h$, write for $\alpha=\sum_{g} \alpha(g) g \in K G, \tilde{\alpha}(g)=\sum_{h \sim g} \alpha(h)$, the sum of coefficients of all conjugates of $g$ in $\alpha$. The following result is well known.

Lemma 1 (Formanex [2]). If $e=\sum e(g) g=e^{2} \in K G$ and $G$ is Noetherian then $\tilde{e}(g)=0$ for $g$ of infinite order.

Now let us suppose that $G / A$ is finite and $A$ is polycyclic. Then the number of infinite cyclic factors in any normal series of $A$ is invariant, called the Hirsch number of $G$. We shall prove Theorem 1 by induction on the Hirsch number of $G$. We shall prove that if $p$ is a prime divisor of $s$ then there is an element $g \in G$ of $p$-power order with $\tilde{e}(g) \neq 0, g \neq 1$.

Suppose that the Hirsch number of $G$ is $\geqslant 1$; then it is easy to see by induction on the solvability length of $A$ that $G$ has a torsion-free normal subgroup $N \neq\{1\}$ and therefore $G / N$ has smaller Hirsch number. Let $\bar{e}$ be the image of $e$ under the natural map $K G \rightarrow K(G / N)$. Then due to Lemma 1 , $\bar{e}(1)=e(1)=r / s$. Therefore, by induction, there is a $\bar{g} \in G / N$ of $p$-power order such that $0 \neq \tilde{e}(\bar{g})=\sum \tilde{e}(h)$, a sum over certain $h$ such that $\bar{h}$ is conjugate to $\bar{g}$.

Since again due to Lemma $1, \tilde{e}(h)=0$ for elements $h$ of infinite order, we have that $\tilde{e}\left(h_{0}\right) \neq 0$ for some $h_{0}$ of finite order. This $h_{0}$ clearly has $p$-power order. Thus it remains to prove

LEMMA 2. If $G$ is finite and $p$ is a prime divisor of $s$ then there exists a $1 \neq g \in G$ of p-power order such that $\tilde{e}(g) \neq 0$.

We shall need

LEMMA 3 (HATTORI [1]). Suppose that $e=\sum e(g) g=e^{2} \in K G$ where $G$ is finite and $K$ has characteristic zero. Let $\chi$ be the character of $G$ afforded by $K G e$. Then for $g \in G$ we have $\left|C_{G}(g)\right| \tilde{e}(g)=\chi\left(g^{-1}\right)$ where $C_{G}(g)$ denotes the centralizer of $g$ in $G$.

Proof. For any $\alpha \in K G$ and $h \in G$, let $T_{\alpha}(h): K G \rightarrow K G$ be the $K$-linear map which sends $y$ to hy $\alpha$. Then $T_{e}(h)$ acts on $K G e$ as left multiplication by $h$ and annihilates $K G(1-e)$. Since $K G=K G e \oplus K G(1-e)$, choosing a suitable basis of $K G$, we see that the trace of the linear transformation $T_{e}(h)$ is equal to $\chi(h)$.

Now $T_{e}(h)=\Sigma_{g} e(g) T_{g}(h)$ and $T_{g}(h)$ sends $x$ to $h x g$ for any $x \in G$. Therefore $T_{g}(h)$ permutes the elements of $G$, so its trace is the number of $x \in G$ with $x=h x g$. But $x=h x g$ if and only if $x^{-1} h^{-1} x=g$. So the trace 
of $T_{g}(h)$ is 0 if and only if $g$ is not conjugate to $h^{-1}$ and is $\left|C_{G}(h)\right|$ otherwise. Hence

$$
\chi(h)=\sum_{g \sim h^{-1}} e(g)\left|C_{G}(h)\right|=\tilde{e}\left(h^{-1}\right)\left|C_{G}(h)\right| .
$$

Proof of Lemma 2. Suppose on the contrary that $\tilde{e}(g)=0$ for all $p$ elements $g \neq 1$. Let $P$ be a Sylow $p$-subgroup of $G$ and let $\chi_{P}$ be the restriction of $\chi$ to $P$. Then $\chi_{P}(g)=0$ for all $1 \neq g \in P$ by Lemma 3. Therefore,

$$
(\chi(1) / \zeta(1)) \zeta(g)=\chi_{P}(g) \text { for all } g \in P,
$$

where $\zeta$ is the character of the regular representation of $P$. Since the 1representation occurs once and only once as a component of the regular representation, it follows that $\chi(1) / \zeta(1)$ is an integer. Thus $\chi(1)$ is a multiple of $|P|$. But $\chi(1)=|G| r / s$, so $p$ cannot divide $s$ and the lemma is proved.

4. Proof of Theorem 2. We may assume (as in [6]) that $R$ is a Noetherian ring without nilpotent elements and therefore is contained in a direct sum of fields; $R \subseteq F_{1} \oplus \cdots \oplus F_{k} \oplus \cdots \oplus F_{r}$ where $F_{i}$ are of characteristic zero for $1 \leqslant i \leqslant k$ and $F_{k+j}$ are of finite characteristic. Let $\Pi_{i}: R G \rightarrow F_{i} G$ be the map induced from the natural projection $R \rightarrow F_{i}$. We claim that $e(1)$ is of the form

$$
e(1)=\left(r / s, r / s, \ldots, r / s, \alpha_{1}, \alpha_{2}, \ldots\right)
$$

where the first $k$ components are equal to the rational number $r / s$. This is true for finite $G$ (see [6]). For polycyclic by finite groups it follows by induction on the Hirsch number in view of Lemma 1. Also, by Zalesskir's Theorem, $\alpha_{i}$ 's belong to finite fields.

We may suppose by considering $1-e$ if necessary that $r / s \neq 0$. Since $r$ and $s$ can be taken to be relatively prime, there exist integers $a$ and $b$ such that $a r+b s=1$. Thus

$$
\beta=a e(1)+b 1_{R}=\left(1 / s, \ldots, 1 / s, a \alpha_{1}+b, a \alpha_{2}+b, \ldots\right) \in R .
$$

We may suppose that $a \alpha_{i}+b \neq 0$ for any $i$, as otherwise a suitable power of $s \beta$ is a nontrivial idempotent in $R$. Now,

$$
s \beta-1=\left(0,0, \ldots, 0, s\left(a \alpha_{1}+b\right)-1, s\left(a \alpha_{2}+b\right)-1, \ldots\right) \in R .
$$

Again, by the same argument, $s\left(a \alpha_{i}+b\right)-1=0$ for all $i$ and so $a \alpha_{i}+b$ $=1 / s$. We have

$$
\beta=(1 / s, \ldots, 1 / s)=1 / s \cdot 1_{R} \in R
$$

Since $s$ is a $|G|$-number as seen by applying Theorem 1 to $\Pi_{1}(e)$, it follows that $s=1$. Therefore, $e=\left(1,1, \ldots, 1, \alpha_{1}, \alpha_{2}, \ldots\right)$. Write $e^{\prime}=1-e$. Then since $\Pi_{i}\left(e^{\prime}\right)$ has trace 0 , it follows that $\Pi_{i}\left(e^{\prime}\right)=0$ for $1 \leqslant i \leqslant k$. Hence $e^{\prime} \in S G$ where $S=F_{k+1} \oplus \cdots \oplus F_{r}$. Let $I$ be the ideal of $R$ generated by the 
coefficients of $e^{\prime}$. Then $I^{2}=I \subset S$. By Krull's theorem, there exists an element $\gamma \in I$ such that $I(1-\gamma)=0$. Thus $\gamma^{2}=\gamma \in R$ and so $\gamma=0$ or 1 . Since clearly $\gamma \neq 1$ as $I \subset S$, we have $\gamma=0$ and hence $I=0$. It follows that $e^{\prime}=0$ and $e=1$.

\section{REFERENCES}

1. A. Hattori, Rank element of a projective module, Nagoya J. Math. 25 (1965), 113-120. MR 31 \#226.

2. E. Formanek, Idempotents in Noetherian group rings, Canad. J. Math. 25 (1973), 366-369. MR 47 \# 5041.

3. M. Parmenter and S. Sehgal, Idempotent elements and ideals in group rings and the intersection theorem, Arch. Math. 24 (1972), 586-600. MR 49 \#350.

4. D. Passman, Infinite group rings, Dekker, New York, 1971. MR 47 \#3500.

5. S. Sehgal, Certain algebraic elements in group rings, Arch. Math. 26 (1975), 139-143.

6. S. Sehgal and H. Zassenhaus, Group rings without non-trivial idempotents, Arch. Math. (to appear).

7. A. Zalesskiǐ, On a problem of Kaplansky, Dokl. Akad. Nauk SSSR 203 (1972), 749-751 = Soviet Math. Dokl. 13 (1972), 449-452. MR 45 \#6947.

Department of Mathematics, University of Alberta, Edmonton, Alberta, Canada t6G 2G1 